\title{
Sliding Mode Fault-Tolerant Controller for Overactuated Electric Vehicles with Active Steering
}

\author{
António Lopes ${ }^{\ddagger *}$, Rui Esteves Araújo ${ }^{\ddagger *}$, António Pedro Aguiar ${ }^{\ddagger \dagger}$ and Maria do Rosário de Pinho \\ ${ }^{*}$ INESC TEC \\ ${ }^{\dagger}$ Research Center for Systems and Technologies \\ †Faculty of Engineering, University of Porto, 4200-465 Porto, PORTUGAL \\ Email:\{antonio.lopes, raraujo, pedro.aguiar,mrpinho\}@fe.up.pt
}

\begin{abstract}
This paper addresses the tracking problem of the state variables of a nonlinear planar dynamic model of an overactuated electric vehicle with four-wheel independent drive (4WID) topology. In order to track the state variables of the system it is proposed a new sliding mode controller based on a nonlinear planar model. The controller explores the overactuated system in order to redistribute the control effort to the remaining actuators when a fault occurs. Although the system has multiple solutions due to the access of the torque applied in each wheel independently, there could be particular fault events where the remaining healthy actuators may not be able to maintain the system stability. In those particular cases the inclusion of the steering control variable is an important advantage as it allows the controller to manipulate the control effort in any directions. The proposed controller is validated in various driving scenarios with different fault schemes. The simulations are carried out with a high-fidelity vehicular model provided by the simulation software Carsim in co-simulation with Matlab/Simulink.
\end{abstract}

\section{INTRODUCTION}

The increasing interest in electric vehicles has posed numerous problems and new challenges on ground vehicle motion control systems. The technological breakthrough in electric vehicles motivated the implementation of different multimotor architectures, unraveling the vast potential of torque vectoring, making vehicles more responsive, controllable and efficient. [1], [2].

One of the most promising multi-motor architectures is the four-wheel independent drive topology, which is defined by four electric actuators that can be controlled separately allowing the definition of the torque in each wheel [1], [3], [2]. Nevertheless, the increase in the number of actuators leads to an increase of fault probability in the system or even total failure. The fault of one actuator compromises the vehicles dynamic control performance when conventional controllers are applied, so the design of the fault-tolerant controllers for electric vehicles is especially important. If the system is able to identify and recover from the initial fault, by restoring the system from a region of deprecated performance to a region of required performance, the complete failure can be avoided [4].

In recent years, the fault-tolerant area have been carefully studied, mostly in aerospace applications where the appearance of faults has a more significant effect and where the physical redundancy of actuators is generalized [5]. Nonetheless, the fault-tolerant control can be applied to other overactuated systems. The ground vehicle is one of the systems where this theme has became more attractive due to implementation of physical and analytical redundancy [1], [5]. The advantages of overactuated system have been studied initially from a stability point-of-view using four-wheel active steering to cope with system disturbance improving handling of the vehicle [6]. An attempt has been made in [1] and its development [7] to improved passive fault tolerant controller based on adaptive control techniques. In addition to improved control methods, the overactuated vehicles allowed the implementation of faulttolerant controllers, particularly in multi-motor topologies [8], [9], [7]. Plenty of solutions have been proposed, from adaptive sliding mode control [10] to model independent adaptive faulttolerant control [9].

The aim of this paper is to present a fault tolerant sliding mode control with active front steering system to hinder the vehicle from spin-out when failures or malfunctions in electric motors occur. The proposed system have an independent control in each wheel and steer-by-wire which allows the modification of the steering information provided by the driver, expanding the control variables in order to maintain a higher order of freedom. This feature presents an important improvement from [2] and enables a more robust fault-tolerant control eliminating points of singularity created by some faults/failures events. In this paper we assumed that the injected faults in the system will only occur in the motors of each wheel and are promptly detected by a fault detection and identification algorithm. This assumption is reasonable according to the literature [7]. The proposed structure of the fault-tolerant control is presented in Fig. 1 which is composed by two sliding mode controllers capable to track perfectly the state variables and a control allocation subsystem that reconfigures the control effort to the available actuators.

The organization of the paper is as follows: Section II formulates the problem of planar vehicle dynamic and state variables tracking in the presence of parametric modeling uncertainty and system disturbance. In section III, the nonlinear control design, the proposed control structure and a solution for sliding mode control design are described. Section IV proposes the control allocation algorithm, and in section $\mathrm{V}$ the performance of the described solution using computer simulation is evaluated. Finally, section VI contains some concluding remarks and presents a final assessment of the presented work. 


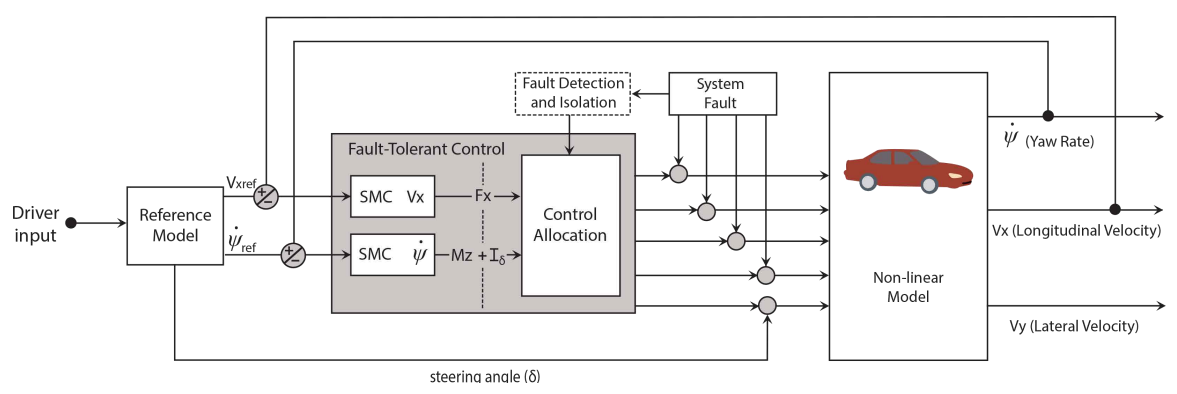

Fig. 1. Proposed control structure with active steering and individual torque control

\section{Control Problem Formulation}

This section describes the planar model of the 4WID ground vehicle using the yaw rate, longitudinal and lateral velocity as system states. The formulation of the tracking problem of the state variables is also discussed in the present section. The control inputs defined for the proposed problem are the forces applied to each of the four wheels and also an active action of steering input of the vehicle.

\section{A. Ground Vehicle Planar Model}

In the planar model it will be neglected the vertical dynamic imposed by the suspension considering the vehicle as a rigid body without any roll or pitch influence [11]. This simplification enables the study of the vehicle frame as an infinitesimal point where all the forces are applied [11].

The vehicle planar dynamic is expressed by two key motions, the translation motion and the rotational motion, that can be defined by the Newton-Euler equations of motion of a rigid vehicle [12].

In this formulation the state variables are the longitudinal and lateral velocity of the vehicle $\left(v_{x}, v_{y}\right)$ and the rate at which the vehicle is rotating over the $\mathrm{z}$ axis, yaw rate $(\dot{\psi})$. The mass of the vehicle is represented by $m$ and the inertial moment of the vehicle in the vertical axis is expressed as $I_{z}$. The variables $F_{x}$ and $F_{y}$ denote the sum of all the external and internal forces applied to the vehicle in the $x$ and $y$ axis respectively, and $M_{z}$ is the total sum of the forces/moment generated in each individual wheel.

By combining the Newton-Euler equations of the vehiclewith the definitions of total moment and lateral force [12] the following planar model arise:

$$
\begin{aligned}
& \dot{v}_{x}=\frac{1}{m}\left(F_{x F R}+F_{x F L}+F_{x R R}+F_{x R L}\right)+\dot{\psi} v_{y} \\
& \dot{v}_{y}=\frac{1}{m}\left(2 C_{f}\left(\delta-\frac{v_{y}}{v_{x}}-\frac{l_{f} \dot{\psi}}{v_{x}}\right)+2 C_{r}\left(\frac{l_{r} \dot{\psi}}{v_{x}}-\frac{v_{y}}{v_{x}}\right)\right)-\dot{\psi} v_{x} \\
& \ddot{\psi}=\frac{1}{I_{z}}\left(2 C_{f} l_{f}\left(\delta-\frac{v_{y}}{v_{x}}-\frac{l_{f} \dot{\psi}}{v_{x}}\right)-2 C_{r} l_{r}\left(\frac{l_{r} \dot{\psi}}{v_{x}}-\frac{v_{y}}{v_{x}}\right)+M_{z}\right)
\end{aligned}
$$

where $C_{F}$ and $C_{R}$ denote the front and rear cornering stiffness respectively, $l_{f}$ and $l_{r}$ express the distance between the front and rear wheels respectively, the inertial moment of the vehicle is defined by $I_{z}$ and the mass of the vehicle is represented by $m$. Furthermore, the steering component $\delta$ can be decomposed in a driver input $\delta_{I N}$ and an active control input $\delta_{u}(\delta=$ $\left.\delta_{I N}+\delta_{u}\right)$.

\section{B. Problem Formulation}

Let $v^{T}=\left[F_{x}, u_{\dot{\psi}}\right]$ be the virtual control variable which will be mapped to real control variables, $u=$ $\left[F_{x F R}, F_{x F L}, F_{x R R}, F_{x R L}, \delta_{u}\right]$, defined by the four forces applied in each wheel and an increment for the steering variable defined by the driver.

Consider the state model of an overactuated ground vehicle, described by (1). Let $e=\left(e_{v_{x}}, e_{\dot{\psi}}\right)=\left(v_{x}-v_{x R e f}, \dot{\psi}-\dot{\psi}_{R e f}\right)$ be the tracking error expressed in the vehicle coordinate frame, where $\left(v_{x r e f}, \dot{\psi}_{\text {Ref }}\right)$ denote the desired reference signal. The goal is to derive a nonlinear control law for the longitudinal force $F_{X}$ and the control variable obtained by the external moment $M_{z}$ and the complementary steering input $\delta_{u}$, based on sliding mode techniques, so that the tracking errors converge asymptotically to zero (or a small ball around zero when not feasible), even in the presence of model uncertainty originated by poor lateral velocity knowledge and/or parametric uncertainty.

The overactuated nature of the system hinders the direct translation of the virtual control variables into the available actuators. To address this fact, we introduce a weighted sum of squares problem with an objective function that pursuit to minimize the total actuator effort [13]. The weight matrix is defined as a diagonal matrix that mirrors the effort required from each actuator and is defined as $W=\operatorname{diag}\left(w_{F L}, w_{F R}, w_{R L}, w_{R R}, w_{\delta_{u}}\right)$ where $w_{i} \in[0,1]$. The control allocation algorithm must set the control variables as a function of the respective virtual control in real time to ensure the optimal solution in each given instant eliminating the effect of actuators faults [14].

\section{Non-LineAR CONTROL DESIGN}

In this section, two decoupled sliding mode control laws to track the lateral and longitudinal motion of the vehicle even in the presence of parametric modeling uncertainty are proposed. The controller is first derived by considering the virtual input control variables $F_{x}$ and $u_{\dot{\psi}}=f\left(M_{z}, \delta_{u}\right)$. It is also assumed that the lateral velocity, although unknown, can be estimated 
through the present model and the error between the estimation and the real value is bounded.

\section{A. Control Structure}

The input introduced by the driver is used to calculate the desirable yaw rate and longitudinal velocity of the vehicle. The controlled steering angle is superimposed to the steering wheel angle resulting into road wheel angle applied to the model. The longitudinal velocity has a much slower dynamic than the yaw rate which allows a decoupled approach to this problem. Thus, the yaw rate and longitudinal velocity are treated independently enabling a decoupled study as well as a separated control of each variable.

\section{B. Sliding Mode Control}

From (1) it is possible to define a strategy for the control design as following: $i$ ) Manipulate the control variable defined by $F_{x}=\left(F_{x F R}+F_{x F L}+F_{x R R}+F_{x R L}\right)$ to regulate the longitudinal velocity $v_{x}$ to the desired value $v_{x R e f}$ and $i i$ ) Actuate on the control variable $u_{\dot{\psi}}$ which forces the vehicle's yaw rate to a desired maneuver. The next result shows the proposed control laws.

Theorem 1 Consider the nonlinear system described by the ground vehicle model (1) in closed-loop with the control law (2a) and (2b).

$$
\begin{aligned}
F_{x} & =\left(\dot{v}_{x R e f}+\dot{\psi} v_{y}+\kappa_{v_{x}}\left(v_{x}, \dot{\psi}\right)\right) m \\
u_{\dot{\psi}} & =-\frac{2 C_{f} l_{f}}{I_{z}} \delta_{I n}+\frac{2 C_{r} l_{f}-2 C_{f} l_{f}}{v_{x} I_{z}} v_{y}+\frac{2 C_{f} l_{f}^{2}-2 C_{r} l_{r}^{2}}{v_{x} I_{z}} \dot{\psi} \\
& +\ddot{\psi}_{\text {Ref }}+\kappa_{\dot{\psi}}\left(v_{x}\right)
\end{aligned}
$$

where $\kappa_{v_{x}}\left(v_{x}, \dot{\psi}\right)$ and $\kappa_{\dot{\psi}}\left(v_{x}\right)$ are non-linear functions described by

$$
\begin{aligned}
\kappa_{v_{x}}\left(v_{x}, \dot{\psi}\right) & =-K_{v_{x}}\left(v_{x}, \dot{\psi}\right) \operatorname{sign}\left(s_{v_{x}}\right) \\
\kappa_{\dot{\psi}}\left(v_{x}\right) & =-K\left(v_{x}\right) \operatorname{sign}\left(s_{\dot{\psi}}\right)
\end{aligned}
$$

where $K_{v_{x}}\left(v_{x}, \dot{\psi}\right)>0$ and $K\left(v_{x}\right)>0$ are control parameters.

The variables $s_{v_{x}}$ and $s_{\dot{\psi}}$ are defined as the error between the state variable and the desired reference.

$$
\begin{gathered}
s_{\dot{\psi}}=\dot{\psi}-\dot{\psi}_{\text {Ref }}=e_{\dot{\psi}} \\
s_{v_{x}}=v_{x}-v_{x R e f}=e_{v_{x}}
\end{gathered}
$$

The motion of the system will be restricted to the manifold $s=0$, for all time $t \rightarrow \infty$, even in the presence of bounded modeling uncertainty.

Proof. The proof is organized as follow: Initially it will be shown that the error of the yaw rate $e_{\dot{\psi}}$ converges asymptotically to zero as $t \rightarrow 0$, assuming the longitudinal velocity remains constant $\left(v_{x} \in \mathbb{R}^{+}\right)$. It will also be shown that the error of the longitudinal velocity $e_{v_{x}}$ converges asymptotically to zero as $t \rightarrow 0$. a) Convergence of yaw rate:

Consider the following Lyapunov candidate:

$$
V=\frac{1}{2} s_{\dot{\psi}}^{2}
$$

Let $x=0$ be an equilibrium point for $\dot{x}=f(x)$ and $D \in$ $\mathbb{R}^{n}$ be a domain containing $x=0$. Let $V: D \rightarrow \mathbb{R}$ be a continuously differentiable function such that:

- $V(0)=0, V(x)>0 ; \forall x \in D\{0\}$

- $\dot{V} \leq 0, \forall x \in D$

Then, $x=0$ is stable. Moreover, if

$$
\dot{V}<0, \forall x \in D\{0\}
$$

then $x=0$ is asymptotically stable, according to Lyapunov's stability theorem [15].

By computing the derivative of $V$ results:

$$
\dot{V}=s_{\dot{\psi}}\left(\ddot{\psi}-\ddot{\psi}_{R e f}\right)
$$

And let's assume, without lost of generality, that the steering variable can be decomposed in two signals $\left(\delta=\delta_{\text {In }}+\delta_{u}\right)$. Applying (1c) to (6) and after some straightforward manipulation, we obtain:

$$
\begin{aligned}
\dot{V}= & s_{\dot{\psi}}\left(\frac{2 C_{f} l_{f}}{I_{z}} \delta_{I n}-\frac{2 C_{r} l_{f}-2 C_{f} l_{f}}{v_{x} I_{z}} v_{y}-\frac{2 C_{f} l_{f}^{2}-2 C_{r} l_{r}^{2}}{v_{x} I_{z}} \dot{\psi}\right. \\
& \left.+\frac{M_{z \text { ext }}}{I_{z}}+\frac{2 C_{f} l_{f}}{I_{z}} \delta_{u}-\ddot{\psi}_{\text {Ref }}\right)
\end{aligned}
$$

From (7) it is possible to identify the control inputs $u_{\dot{\psi}}$, given by:

$$
u_{\dot{\psi}}=\frac{M_{z e x t}}{I_{z}}+\frac{2 C_{f} l_{f}}{I_{z}} \delta_{u}
$$

This control variable $u_{\dot{\psi}}$ is now defined as presented in (2b). Substituting (2b) in (7) results:

$$
\dot{V}=s_{\dot{\psi}}\left(-\frac{2 C_{r} l_{f}-2 C_{f} l_{f}}{v_{x} I_{z}} \tilde{v}_{y}+\kappa_{\dot{\psi}}\left(v_{x}\right)\right)
$$

where $\tilde{v}_{y}$ denote the error of the estimated lateral velocity, which it is assumed bounded by

$$
\left|-\frac{2 C_{r} l_{f}-2 C_{f} l_{f}}{v_{x} I_{z}} \tilde{v}_{y}\right| \leq \varrho\left(v_{x}\right)
$$

Let $\kappa_{\dot{\psi}}\left(v_{x}\right)=-K\left(v_{x}\right) \cdot \operatorname{sign}\left(s_{\dot{\psi}}\right)$ where $K\left(v_{x}\right) \geq \varrho\left(v_{x}\right)+$ $K_{0}, K_{0}>0$ Then

$$
\begin{aligned}
& \dot{V} \leq s_{\dot{\psi}}\left(\varrho\left(v_{x}\right)-K\left(v_{x}\right) \cdot \operatorname{sign}\left(s_{\dot{\psi}}\right)\right) \\
& \dot{V} \leq\left|s_{\dot{\psi}}\right|\left(\varrho\left(v_{x}\right)-K\left(v_{x}\right)\right) \\
& \dot{V} \leq-K_{0}\left|s_{\dot{\psi}}\right|
\end{aligned}
$$

The inequality (11) guarantees the final condition to the asymptotic convergence imposed by the Lyapunov's stability theorem, proving that the yaw rate error converges asymptotically to zero as $t \rightarrow \infty$. 
b) Convergence of longitudinal velocity:

Using the Lyapunov candidate expressed in (5) the first condition for asymptotic convergence is guaranteed. Computing the time derivative of the candidate results:

$$
\dot{V}=s_{v_{x}}\left(\dot{v}_{x}-\dot{v}_{x R e f}\right)
$$

The longitudinal dynamic expressed in (1a) is substituted in (12), yielding

$$
\begin{aligned}
\dot{V} & =s_{v_{x}}\left(\frac{1}{m}\left(F_{x F R}+F_{x F L}+F_{x R R}+F_{x R L}\right)\right. \\
& \left.+\dot{\psi} v_{y}+\gamma\left(v_{x}\right)-\dot{v}_{x R e f}\right)
\end{aligned}
$$

where $\gamma\left(v_{x}\right) \geq 0$ is the bounded resistance forces that acts in the vehicular system, such as the friction forces acting in each individual tire and the drag resistance force [11].

$$
\left|\frac{1}{m}\left(4 . F_{R}+\frac{1}{2} \rho v_{x}^{2} C_{D} A\right)\right| \leq \gamma\left(v_{x}\right)
$$

The total control effort can be expressed as the sum of the forces applied at each wheel as $\left(F_{x}\right)$. The design control law defined in (2a) is replaced in (13) resulting:

$$
\dot{V}=s_{v_{x}}\left(\dot{\psi} \tilde{v}_{y}+\gamma\left(v_{x}\right)+\kappa_{v_{x}}\left(v_{x}, \dot{\psi}\right)\right)
$$

Assume once again that the error of the lateral velocity estimation is bounded and the total system uncertainty is bounded as $\xi\left(v_{x}, \dot{\psi}\right) \geq\left|\dot{\psi} \cdot \tilde{v}_{y}+\gamma\left(v_{x}\right)\right|$. By defining $\kappa_{v_{x}}\left(v_{x}, \dot{\psi}\right)=$ $-K_{v_{x}}\left(v_{x}, \dot{\psi}\right) \operatorname{sign}\left(s_{v_{x}}\right)$ with $K_{v_{x}}\left(v_{x}, \dot{\psi}\right) \geq \xi\left(v_{x}, \dot{\psi}\right)+K_{v_{x} 0}$, and $K_{v_{x} 0}>0$, results the condition (16)

$$
\begin{aligned}
& \dot{V} \leq s_{v_{x}}\left(\xi\left(v_{x}, \dot{\psi}\right)-K_{v_{x}}\left(v_{x}, \dot{\psi}\right) \operatorname{sign}\left(s_{v_{x}}\right)\right) \\
& \dot{V} \leq\left|s_{v_{x}}\right|\left(\xi\left(v_{x}, \dot{\psi}\right)-K_{v_{x}}\left(v_{x}, \dot{\psi}\right)\right) \\
& \dot{V} \leq-K_{v_{x} 0}\left|s_{v_{x}}\right|
\end{aligned}
$$

The two conditions of asymptotic stability were successfully proven through the Lyapunov candidate (5) and the resulting condition (16), thus proving the stability of both proposed control laws to the planar vehicle system.

\section{Control Allocation}

In Fig. 1 it is clear the presence of the control allocation block that is designed to distribute the effort of the actuators in an optimal way and to eliminate the disturbance created by the actuators faults. The virtual control $v$ introduced in Section II is mapped into the physical actuators through least squares regression obtaining an overall solution that minimizes the sum of the squares of the errors. In this particular case the system can have more than one solution, so there is a weighting factor imposed to each variable which reflects the significance of that variable. This paper proposes a dynamic weighting matrix that expresses the importance given to each actuator in a particular moment, redefining the weighting matrix according to the system conditions (system faults, wheel workload, etc). In this analysis it is assumed that the system faults and failures are tackled by a fault detection and identification system, providing this information to the proposed controller.

\section{A. Weighting Matrix}

The weighting matrix $\mathrm{W}$ is chosen in order to:

- Redistribute the control in the presence of faults in the system;

- Explore the tire load to improve the grip.

Considering that the lateral forces that the tire originates are negligible $\left(F_{x i j} \gg F_{y i j}\right)$, the workload of each tire can be expressed as [10], [13]

$$
\begin{aligned}
\eta_{F L} & =\frac{F_{x F L}}{\mu F_{z F L}} ; & \eta_{F R} & =\frac{F_{x F R}}{\mu F_{z F R}} ; \\
\eta_{R L} & =\frac{F_{x R L}}{\mu F_{z R L}} ; & \eta_{R R} & =\frac{F_{x R R}}{\mu F_{z R R}} ;
\end{aligned}
$$

A weighting matrix $J$ regarding the tire workload can be expressed as

$$
J=\operatorname{diag}\left(\left(\mu \bar{F}_{z F L}\right)^{2},\left(\mu \bar{F}_{z F R}\right)^{2},\left(\mu \bar{F}_{z R L}\right)^{2},\left(\mu \bar{F}_{z R R}\right)^{2}, 1 / 100\right)
$$

where $\bar{F}_{z i j}$ is the normalized value of the vertical force expressed by:

$$
\bar{F}_{z i j}=\frac{F_{z i j}}{\max \left(F_{z i j}\right)}
$$

The matrix $J$ in (18) is a diagonal weighting matrix, where $j_{i i} \leq 1, i \in\{1,2,3,4,5\}$. The weight defined for the steering increment $j_{55}$ is $\times 100$ lower then the remaining variables and relieves the effort applied to the steering component.

In order to express the effect of actuator faults, it will be assumed that the occurrence of a fault has a direct impact on the available torque of the actuator. Considering the matrix $K$ that represents the actuator fault expressed as

$$
K=\operatorname{diag}\left(k_{F L}, k_{F R}, k_{R L}, k_{R R}, k_{\delta}\right)
$$

with $k_{i j} \in[0,1]$, where $k_{i j}$ expresses the lost of effectiveness of the $i j$ th actuator. Then it is possible to define a weighting matrix $M$ that exploit the actuator effectiveness

$$
M=(I-K)
$$

where $I$ is the identity matrix.

The final weighting matrix $W$ can be obtained by combining the $M$ matrix (21) relative to the system faults with the load restriction (17) obtaining:

$$
\begin{aligned}
W= & \operatorname{diag}\left(\left(1-k_{F L}\right)\left(\mu \bar{F}_{z F L}\right)^{2},\left(1-k_{F R}\right)\left(\mu \bar{F}_{z F R}\right)^{2},\right. \\
& \left.\left(1-k_{R L}\right)\left(\mu \bar{F}_{z R L}\right)^{2},\left(1-k_{R R}\right)\left(\mu \bar{F}_{z R R}\right)^{2}, k_{\delta} / 100\right)
\end{aligned}
$$

\section{B. Weighted sum of Squares}

The virtual control variable is the result of a linear combination of control effort in each actuator:

$$
G u=v
$$

In this particular problem, the $G$ matrix is a non-square singular matrix, where the number of columns of the matrix (n) is greater than the number of lines $(\mathrm{m})(m=2 n=5)$, which means that the problem is under determined. This 
characteristic emerges from the fact that we are trying to map the virtual control variables $\left(v \in \mathbb{R}^{2}\right)$ into five physical actuators $\left(u \in \mathbb{R}^{5}\right)$. Nonetheless, it is possible to define the following transformation:

$$
u=G^{\dagger} v
$$

where $G^{\dagger}$ is the right pseudo-inverse of $G$. One choice of $G^{\dagger}$ is obtained from the following minimization problem [5], [10]:

$$
\begin{gathered}
\min _{u} u^{T} W^{-1} u \\
\text { subjected to } G u=v
\end{gathered}
$$

$W \in \mathbb{R}^{n \times n}$ is a symmetric positive definite diagonal weighting matrix [5]. This minimizes the cost associated with the control vector $u$ at each time instant. The optimal solution to (25) is presented as [5], [10]:

$$
u=\left[W G^{T}\left(G W G^{T}\right)^{-1}\right] v
$$

\section{Simulation Results}

To illustrate the performance of the control scheme proposed in Fig. 1 to parametric model uncertainty, actuators faults or even total failure, a series of tests were carried out with help of a high-fidelity simulator, the CarSim in co-simulation with Matlab/Simulink. In order to demonstrate the fault-tolerant control capability it is defined two maneuvers: the J-turn maneuver with constant acceleration and Double Lane Change maneuver with constant longitudinal velocity.

\section{A. J-turn}

In the first maneuver the vehicle starts at the origin $(0,0)$ with an initial longitudinal velocity of $45 \mathrm{Km} / \mathrm{h}$ and is moving in a straight line with null yaw rate. At the time $t=1 \mathrm{~s}$ it is enforced a ramp longitudinal velocity reference with a constant slope until it reaches $60 \mathrm{Km} / \mathrm{h}$. The turning maneuver starts at the time $t=2 s$ and continues for the remaining time of the simulation. In order to evaluate the performance of the controller to complete failure of multiple actuators, it is imposed a double failure of the two left wheel actuator's (Front and Rear) at time $t=4 s$.

From the analysis of the result in Fig. 2 it is possible to see that the global trajectory of the vehicle is significantly affected by the failure of both actuators. On the other hand, the proposed controller is able to maintain the system stability even in the presence of multiple actuators failure. The torque applied at each individual wheel and the steering input variable is presented in Fig. 3.

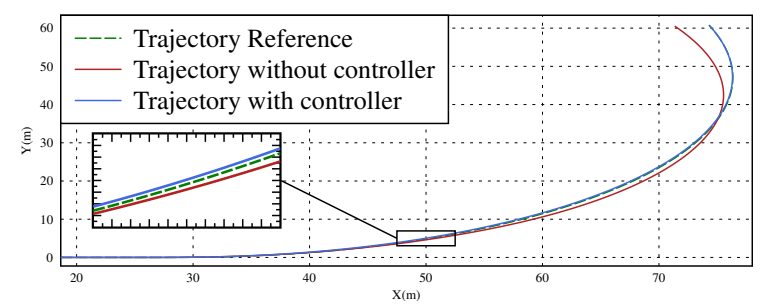

Fig. 2. Global position of the vehicle in J-turn
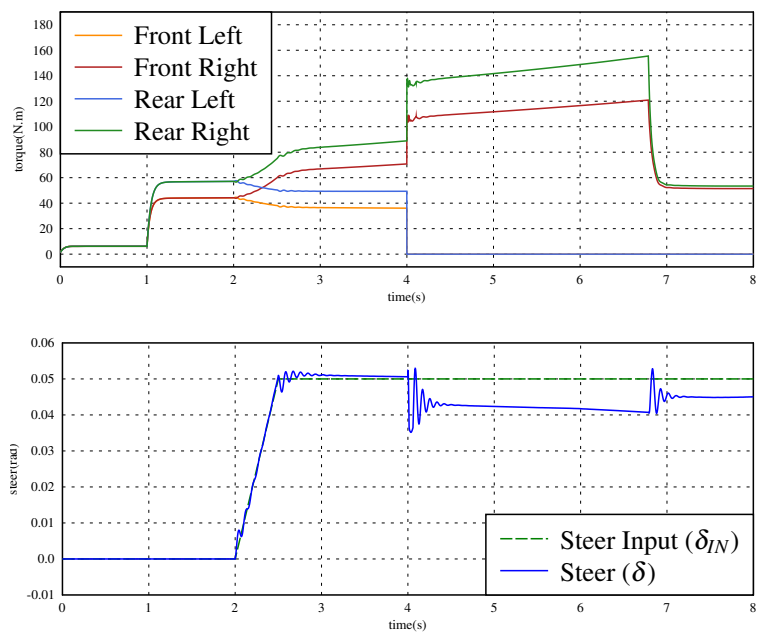

Fig. 3. Control variables in J-turn a)Torque applied in each wheel b)steering

In the instant $t=4 \mathrm{~s}$ the amount of torque applied in the left wheels becomes null, as they suffer a simultaneous complete failure. However, the proposed controller is able to reconfigure and redistribute the total control effort in order to maintain the longitudinal velocity of the vehicle by increasing the torque applied in the right wheels. From this fault scheme rise another fundamental problem, the external moment $M_{\text {zext }}$ applied to the vehicle can not be controlled as the two healthy actuators are at the same side of the vehicle which means that there is no degrees of freedom to actuate in the lateral dynamic of the vehicle. In this situation, the steering component is the only control variable that can control the yaw rate of the system. As seen in Fig. 3(b) the steering input of the system adapts in order to maintain the trajectory of the vehicle, and so reestablishing the stability of the system.

\section{B. Double Lane Change}

The Double Lane Change is a more demanding maneuver that will be carried out at constant speed $\left(v_{x}=90 \mathrm{Km} / \mathrm{h}\right)$ and starts with a null yaw rate, heading straight until $t=$ $0.8 s$. In this simulation it is introduced three actuators faults: a complete failure of the actuator at the front left wheel at time $t=4 s$, a $60 \%$ lost of effectiveness in the rear right wheel at the instant $t=6 \mathrm{~s}$ and another complete failure of the actuator at the rear left wheel at $t=8 s$.

The state variables yaw rate and longitudinal velocity are represented in the Fig. 4 . We can see that the vehicle converges to the desired references, even in the presence of multiple actuators faults. The velocity error presented in Fig. 4(b) results from a quasi-sliding switching function.

Fig. 5(a) expresses the torque applied in each individual wheel, once again, the proposed controller redistributes the control effort through the remaining actuators. At the instant $t=4$ the lost of the effectiveness of the actuator in the front left wheel is promptly compensated by increasing the torque of the rear left wheel actuator and with that maintain the total longitudinal force applied to the vehicle. Furthermore, there is 

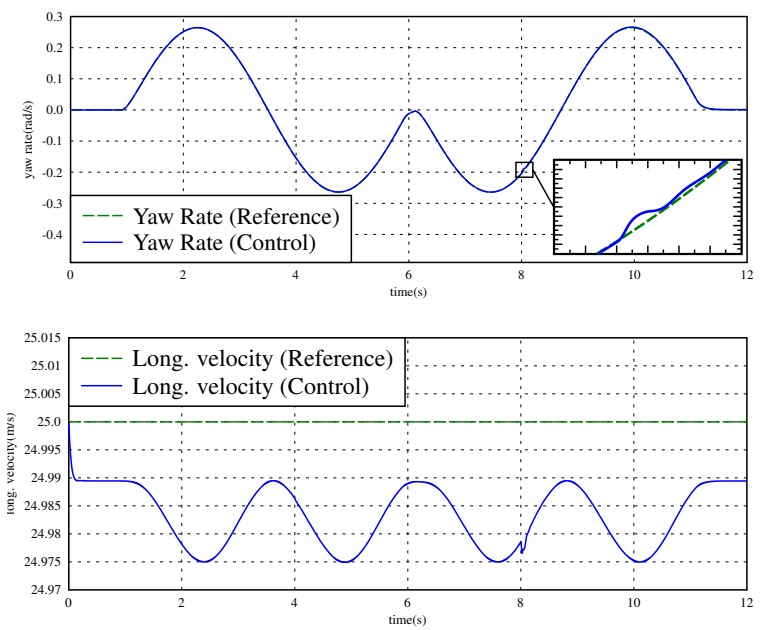

Fig. 4. State Variables in DLC a)Yaw Rate b)longitudinal velocity
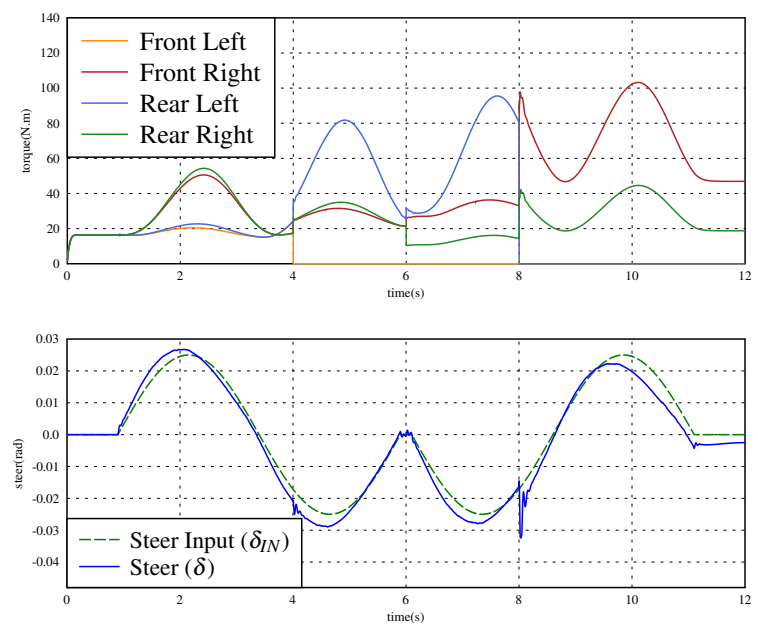

Fig. 5. Control Variables in DLC a)Torque applied in each wheel b)steering

a distinction between the torque applied in each wheel since the very beginning of the maneuver. This is a direct result of the proposed optimal distribution that target the wheels with most vertical force applied. From the time instant $t=0.8$ (beginning of the DLC maneuver) until time $t \approx 3.5$ the vehicle is curving to the left, as a result, there is a lateral acceleration that ultimately result in a difference of vertical force between the right and the left tires. It is also possible to identify a small difference between the front and rear tires, that is originated,by the difference of vertical load in each wheel. This optimal allocation promotes the system stability by targeting the wheel that is less likely to suffer slip.

By examining the result expressed on Fig. 5(b) it is possible to conclude that, although the system faults at time $t=4 \mathrm{~s}$ and $t=6 \mathrm{~s}$ perturb the steering variable, it is just in the failure, at time $t=8 \mathrm{~s}$, that the steering variable as a significant action and becomes most valuable. Just like in the previous simulation, at time $t=8 \mathrm{~s}$, the external moment defined by the longitudinal forces applied to the wheel is no longer available and it becomes essential to take an additional action over the steering variable.

\section{CONCLUSION}

The paper addressed the problem of the planar dynamic of the overactuated ground vehicle, in the presence of parametric modeling uncertainty and actuators fault. The proposed solution uses a decoupled double sliding mode controller in order to promote stability to the two state variables yaw rate and longitudinal velocity. The paper validates the controller for a four wheel independent drive topology including an active steering control variable that promotes stability to the system, particularly in situations where a singularity arise from the fault scheme.

In future research it will be proposed a state observer in order to obtain a more reliable information of the vehicle side slip therefore eliminating the disturbance originated in the system. The inclusion of other system faults on the steering actuator and the fault detection and identification system will also be introduced in the near future.

\section{REFERENCES}

[1] R. Wang and J. Wang, "Fault-tolerant control with active fault diagnosis for four-wheel independently driven electric ground vehicles," Vehicular Technology, IEEE Transactions on, vol. 60, no. 9, pp. 4276-4287, 2011.

[2] A. Lopes and R. E. Araújo, "Fault-tolerant control based on sliding mode for overactuated electric vehicles," in Electric Vehicle Conference (IEVC), 2014 IEEE International. IEEE, 2014.

[3] R. de Castro, , M. Tanelli, S. M. Savaresi, and D. Freitas, "Torque blending and wheel slip control in evs with in-wheel motors," Vehicle System Dynamics, vol. 50, no. sup1, pp. 71-94, 2012.

[4] M. Blanke, M. Kinnaert, J. Lunze, M. Staroswiecki, and J. Schrder, Diagnosis and fault-tolerant control. Springer Publishing Company, Incorporated, 2010.

[5] H. Alwi, C. Edwards, and C. P. Tan, Fault detection and fault-tolerant control using sliding modes. Springer Science \& Business Media, 2011.

[6] N. Hamzah, Y. M. Sam, H. Selamat, M. K. Aripin, and M. F. Ismail, "Yaw stability improvement for four-wheel active steering vehicle using sliding mode control," in Signal Processing and its Applications (CSPA), 2012 IEEE 8th International Colloquium on. IEEE, 2012, pp. 127-132.

[7] R. Wang and J. Wang, "Fault-tolerant control for electric ground vehicles with independently-actuated in-wheel motors," Journal of Dynamic Systems, Measurement, and Control, vol. 134, no. 2, p. 021014, 2012.

[8] X. Zhang, V. Cocquempot, B. Jiang, and H. Yang, "Active fault diagnosis based on fault-tolerant control with control constraints for an electric 4wd vehicle," in Control and Automation (ICCA), 2013 10th IEEE International Conference on. IEEE, 2013, pp. 1724-1729.

[9] D.-Y. Li, Y.-D. Song, D. Huang, and H.-N. Chen, "Model-independent adaptive fault-tolerant output tracking control of 4ws4wd road vehicles," Intelligent Transportation Systems, IEEE Transactions on, vol. 14, no. 1, pp. 169-179, 2013.

[10] K. Nam, H. Fujimoto, and Y. Hori, "Design of an adaptive sliding mode controller for robust yaw stabilisation of in-wheel-motor-driven electric vehicles," International Journal of Vehicle Design, vol. 67, no. 1, pp. 98-113, 2014

[11] M. Abe, Vehicle handling dynamics: theory and application. Butterworth-Heinemann, 2015.

[12] R. N. Jazar, Vehicle dynamics: theory and application. Springer Science \& Business Media, 2013.

[13] N. Ando and H. Fujimoto, "Yaw-rate control for electric vehicle with active front/rear steering and driving/braking force distribution of rear wheels," in Advanced Motion Control, 2010 11th IEEE International Workshop on. IEEE, 2010, pp. 726-731.

[14] E. Ono, Y. Hattori, Y. Muragishi, and K. Koibuchi, "Vehicle dynamics integrated control for four-wheel-distributed steering and four-wheeldistributed traction/braking systems," Vehicle System Dynamics, vol. 44, no. 2 , pp. 139-151, 2006.

[15] H. K. Khalil and J. Grizzle, Nonlinear systems. Prentice hall New Jersey, 1996, vol. 3. 